\title{
MARGINAL ACCURACY OF CAD/CAM FABRICATED MONOLITHIC ALL-CERAMIC CROWNS AS A FUNCTION OF DIGITAL SCANNING TECHNIQUES
}

\author{
Rasha Sayed Asaad*
}

\begin{abstract}
Purpose : The aim of this study was to evaluate the influence of intraoral and extraoral scanning techniques on the vertical marginal gap distance of two types of computer aided designing/computer aided manufacturing (CAD/CAM) fabricated all-ceramic monolithic crowns. Materials and methods: Maxillary and mandibular typodont models were used in this study in which a standardized all-ceramic preparation of lower first molar tooth was carried out. These typodont models with the prepared tooth were representing the patient's mouth. Thirty all-ceramic crowns were fabricated by CAD/CAM technique and divided into three groups (10 crowns each) according to their scanning technique; Group I: scanning of the typodont models by intraoral scanner. Group II: scanning of silicon impressions by extraoral scanner. Group III : scanning of stone casts by extraoral scanner. Each group was further sub-divided into 2 sub-groups ( 5 crowns each) according to the type of crown material. Sub-group(A): zirconia(BruxZir) crowns and Sub-group (B): hybrid ceramic(Vita Enamic) crowns. The vertical marginal gap distance between each crown margin and finish line of the prepared tooth was measured using a Digital microscope of 40X at 16 points / crown. Data were collected, tabulated and statistically analyzed using Tukey's post-hoc and Two-way ANOVA tests $(\mathrm{P}<0.05)$. Results: Different scanning techniques as well as crown materials had a statistically significant effect on vertical marginal gap distance $(\mu \mathrm{m})$ at $\mathrm{P}$-value 0.026 . The intraoral scanning technique recorded the lowest statistically significant vertical marginal gap distance mean values for both crown materials among the three scanning techniques. Regarding the crown material, hybrid ceramic Vita Enamic crowns had a lower statistically significant vertical marginal gap distance values than zirconia BruxZir crowns with all the tested scanning techniques. Conclusion: The vertical marginal gap distance of monolithic crowns was significantly affected by the scanning techniques and crown materials with the superiority of the intraoral scanning technique. Vita Enamic crowns had lower vertical marginal gap distance than BruxZir crowns with different scanning techniques. All the tested monolithic crowns had vertical marginal gap distance values within the clinically acceptable levels.
\end{abstract}

KEY WORDS: intraoral scanner, extraoral scanner, vertical marginal gap distance, monolithic, hybrid ceramics, Vita Enamic, zirconia, BruxZir.

* Oral and Maxillofacial Prosthodontic Department, Faculty of Dentistry, King Abdulaziz University, Jeddah, Kingdom of Saudi Arabia 


\section{INTRODUCTION}

Advanced ceramics such as zirconia have shown to be appropriate substitutes for dental clinical applications. Zirconia has been used as an aesthetic restorative material due to its excellent properties which include a high flexural strength, a superior fracture resistance and an ideal color stability ${ }^{[1,2]}$. Nevertheless, New restorative materials have been developed seeking a more realistic mimicking of the natural dental structures. These materials include polymer infiltrated ceramics (PICs) with a dual network structure. This hybrid ceramic consists of two phases; the porous feldspathic ceramic (75 vol\%) and the reinforcing phase of acrylic polymer (25 vol\%). ${ }^{[3]}$ According to Min et al. (2016) ${ }^{[4]}$ PICs have mechanical properties that are superior to those of pure ceramics, enabling stability against masticatory forces, as PICs prevent the propagation of cracks, due to the interpenetration of polymer in the material.

High mechanical performance, perfect marginal fit, and excellent esthetics are considered the utmost crucial needs for the construction of fixed prostheses .To attain these requirements, (CAD/ CAM) technology has been developed recently, allowing the standardized manufacturing of greatly accurate monolithic crowns with a better congruent composition and less flaws or voids in comparison to their long established porcelain veneered crowns. $^{[5]}$ Additionally, the utilization of precision milling and exclusion of various firing steps required for fabricating all-ceramic prostheses, have reduced the time, manufacturing steps and cost of the fabrication process of CAD/CAM ceramics. ${ }^{[6]}$

Several clinical studies have reported that the accuracy of any prosthesis particularly in the marginal area plays an important role for successful long term clinical performance as any marginal discrepancy can lead to secondary caries, periodontal problems, and finally prostheses failure. ${ }^{[7,8]}$
In dentistry, the impression step is the most important one in the fabrication of dental prostheses. The conventional impression technique include many steps: teeth preparation, tissue retraction, impression taking, pouring into master cast, wax up and finally casting, so there are numerous items could affect the precision of the final restoration. Also the conventional impression has a lot of drawbacks as some patients can feel discomfort and/or have gaging reflex during impression taking and finally errors due to incorporation of air bubbles which can cause inaccuracies in the stone model. Subsequently, a great improvement recently has occurred in impression techniques to overcome all of these limitations. ${ }^{[9]}$ In comparison to the conventional method, the digital method eliminates clinical steps as: tray selection, impression material setting and disinfection and laboratory steps as: pouring, mounting, sectioning, and the fabrication of the restoration. ${ }^{[10]}$

Recently, many companies innovated highly accurate scanners with advanced software with advanced technology that allow taking of three dimensional (3D) virtual images of the prepared teeth intra-orally. These scanners begin to be considerably used in clinical dentistry to fabricate digital models without taking a conventional impression, then the prostheses were designed using CAD software depends on the taken data that serves as a virtual wax-up. The introduction of digital scanning systems have improved the marginal accuracy of obtained restorations as a consequence of obtaining precise final impression. ${ }^{[1,12]}$

Incorporation of $\mathrm{CAD} / \mathrm{CAM}$ technology to dentistry help to deal with the restrictions found in the conventional impression techniques as they allow rapidity and accuracy of storing scanned images without any distortion. Also, a lot of patients consider digital impressions to be easier and more comfortable technique in comparison to conventional impression techniques. ${ }^{[13]}$ In the construction 
of a restoration using a CAD/CAM technology a lot of aspects could influence marginal adaptation as: design of preparation, marginal location (supragingival or subgingival), designing software, scanning systems, quality of milling machine, and milling tool. ${ }^{[14,15]}$

Several CAD/CAM systems permit the design and fabrication of prostheses through scanning classic impression itself or stone casts after pouring the impression using extraoral scanners which can take more than one visit. However, in fabrications involving classic impression or a stone cast, a possibility of technical mistakes can exist that can affect the standard of final prosthesis. ${ }^{[15]}$ Recently, digital impressions using intraoral scanners have become widely used to fabricate digital models of oral structures; these digital impressions are used in the construction of CAD/CAM restorations, making the fabrication process easier that allow tooth preparation and crown cementation in single visit. ${ }^{[14,16]}$

The development of high precision restorations became one of the major essential factors in the development of CAD/CAM systems. ${ }^{[17]}$ Recent researches have proven that the precision of $\mathrm{CAD} /$ CAM restorations mostly presented marginal gaps within the clinically acceptable parameters, comparable with, or even better than those obtained by conventional lost-wax methods. ${ }^{[10,17,18]}$ A completely digital workflow can be achieved with intraoral scanners and digital impressions. Studies have proclaimed that this technology not only eliminated potential problems related to conventional impressions, but also allowed the operator and technician to view the preparation simultaneously, and discuss its main problems. The accuracy of digital impressions has been the aim of different studies in which restorations produced from digital impressions showed marginal gap values similar to, or significantly lower than those obtained from conventional impressions ${ }^{[19-21]}$. However, a lot of clinical trials have declared that single-tooth restorations fabricated in a completely digitized workflow have a fitting quality that is equal to or better than conventionally fabricated restorations. ${ }^{[22,23]}$ Moreover, compared with conventional impressions, digital impressions can be more simple, accurate ,accepted by patients, time-efficient and finally can improve the treatment outcomes. ${ }^{[24,25]}$

Studies that predict the clinical behavior of newly engineered materials, like PICs, are scarce when comparing with, Zirconia-based ceramic materials. In this study, the performance of the prosthetic materials zirconia and polymer infiltrated ceramic (PIC) known as Vita Enamic were compared and investigated in terms of marginal accuracy.

The aim of this study was to evaluate the influence of intraoral and extraoral scanning techniques on the vertical marginal gap distance of two different all-ceramic monolithic crowns. The null hypothesis for this study was that no differences could exist between zirconia (BruxZir) and hybrid ceramic (Vita Enamic) crowns in vertical marginal gap distance fabricated through three different scanning techniques.

\section{MATERIALS AND METHODS}

\section{Samples grouping:}

Maxillary and mandibular typodont models (Frasaco GmbH, Tettnang, Germany) were used in this study in which a standardized all-ceramic preparation of lower first molar tooth was carried out with deep chamfer finish line $(1 \mathrm{~mm}), 2 \mathrm{~mm}$ occlusal reduction, 1.5 axial reduction, $(4 \mathrm{~mm})$ minimum preparation height and convergence angle $\left(6^{\circ}\right)$. [26] These typodont models with the prepared tooth were representing the patient's mouth. Thirty all-ceramic crowns were fabricated and divided into three groups (10 crowns each) according to their scanning technique: Group I: scanning of the typodont models by intraoral scanner. Group II: scanning of silicon impressions by extraoral scanner. Group III : scanning of stone 
casts by extraoral scanner. Each group was further sub-divided into 2 sub-groups (5 crowns each) according to the type of crown material. Sub-group (A): zirconia (Translucent zirconia blanks BruxZir ${ }^{\mathrm{TM}}$ shaded blanks, Glidewell, Frankfurt, Germany ) and sub-group (B): hybrid ceramic (Vita Enamic (B)VITA Zahnfabrik,Bad Sackingen, Germany)

\section{A-Digital Scanning Techniques:}

Scanning of typodont models with the prepared tooth:(representing the patient's mouth) [Intraoral scanning]

The maxillary and mandibular typodont models with prepared tooth were digitally scanned (ten scans) by an intraoral scanner (Trios 3, 3 shape, Copenhagen, Denmark) with a powder-free technology. Fig.(1) The scanner was held closely over the tooth. The TRIOS system is an open system that can export 3D data as a (Standard Tessellation Language) STL file. The resulting scans were then converted to STL format and sent directly to the lab. At the dental lab, the digital data from the scanning process was used to create virtual models using CAD system software (Dental system 2016 v 1.6.3, 3Shape, Copenhagan, Denmark). After exploring the STL files, the designing process for the fullcontour monolithic crowns was performed with the CAD software. The minimum material thickness was set to $1 \mathrm{~mm}$ and the spacer thickness was set to $40 \mu \mathrm{m}$. Data were transported to the computer connected to the milling machine to analyze and start milling full-contour monolithic Zirconia and Vita Enamic crowns.

\section{Extraoral scanning of the silicon impressions:}

A single-step, double consistency ten full-arch addition silicon impressions of the typodont models were made by injecting light body material (Elite, Zhermack, Italy) after being auto-mixed over the prepared tooth and the heavy body addition silicon was also auto-mixed and injected to fill the tray, then a custom tray was seated over the model. The silicon impressions were removed from the typodont model after complete setting according to the manufacturer's instructions, sprayed with surface disinfectant, and rinsed. Then a non-aqueous developer spray (SKD-S2, Spotcheck, Magnaflux, USA) was applied to the impression surfaces. ${ }^{[27]}$ Finally, they underwent extraoral scanning using a laboratory scanner (E2 Lab scanner, 3 shape, Copenhagen, Denmark) that include 5MP camera, Blue LED and multi-line scanning for best detail reproduction. Fig. (2) The resulting scans were then converted to STL format and sent directly to the lab. The designing and milling processes were performed similar to group I.

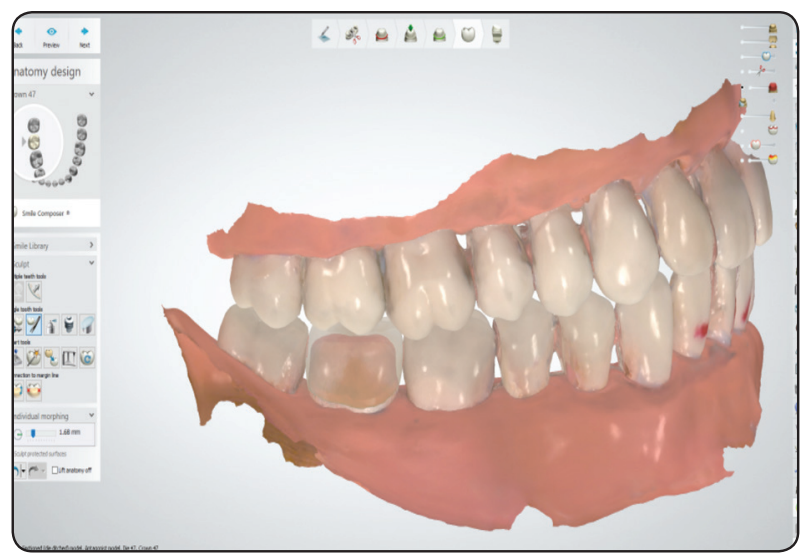

Fig. (1) Intraoral scanning of upper and lower typodont models

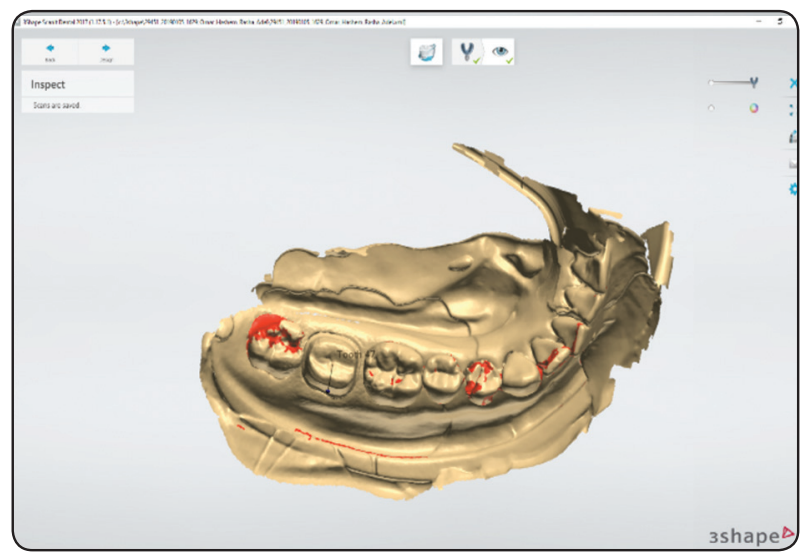

Fig. (2) Extraoral scanning of the silicon impression 


\section{Extraoral scanning of stone casts:}

A single-step full-arch ten silicon impressions (Elite, Zhermack, Italy) were taken and disinfected as described before. After 30 minutes, each impression was poured in Type IV dental stone (Shera premium type IV, SHERA, Germany) by the same technician manually following the manufacturer's recommendations on a vibrator to obtain ten stone casts free from any voids. Then after setting of the stone (16 min), the casts were separated from the impressions, sprayed by Telescan spray (DFS-DIAMON,GmbH) and then scanned using the same laboratory scanner (E2 Lab scanner, 3 shape, Copenhagen, Denmark) Fig.(3). Designing and milling processes were performed similar to group I.

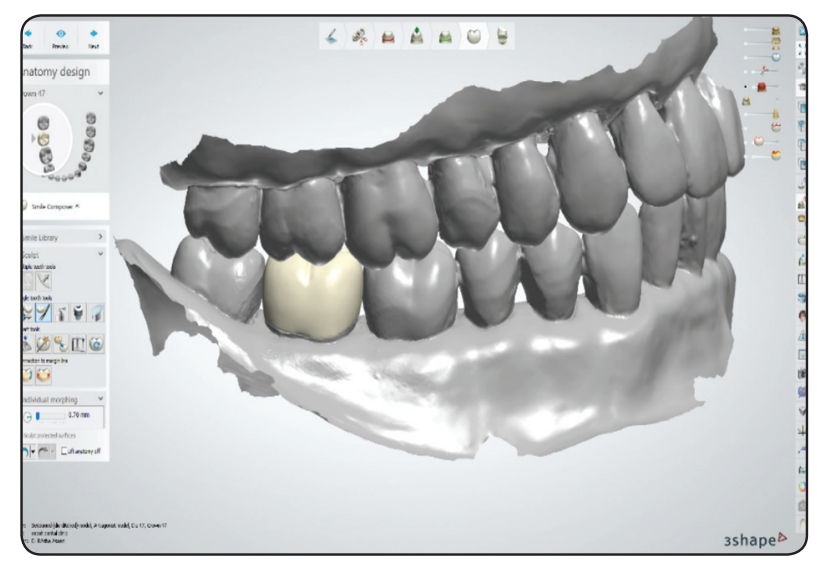

Fig. (3) Extraoral scanning of upper and lower stone casts

\section{B- Construction of Monolithic All-Ceramic Crowns}

The crowns design was manipulated by Dental system software (2016v 1.6.3, 3Shape, Copenhagan, Denmark) and sent to the milling machines to fabricate monolithic Zirconia (BruxZir ${ }^{\mathrm{TM}}$, Glidewell, Frankfurt, Germany) and Vita Enamic crowns ${ }^{\circledR}$ (VITA Zahnfabrik, Bad Sackingen, Germany)

\section{Construction of Monolithic Zirconia Crowns:}

Translucent zirconia blanks ( BruxZir ${ }^{\mathrm{TM}}$ shaded blanks, Glidewell, Frankfurt, Germany ) were used in this study which is composed of ( Zirconium Oxide $\mathrm{ZrO}_{2}<89 \%$, Yttrium Oxide $\mathrm{Y}_{2} \mathrm{O}_{3}>6 \%$, Hafnium Oxide $\mathrm{HfO}_{2}>4 \%$, Aluminum Oxide $\mathrm{Al}_{2} \mathrm{O}_{3}>1 \%$ )

\section{i) Milling of Zirconia Crowns:}

Zirconia blanks were inserted into (Roland DWX-51D, Japan) milling machine, which is a 5-axis milling machine. The crowns were milled with an 25\% enlargement. Each blank is labeled with a barcode that is automatically read by the milling machine to calculate the exact oversize needed during milling to compensate for the sintering shrinkage. After the milling process finished a specific BruxZir finishing bur was used to separate the crowns from the blank. All crowns were then put in an ultrasonic bath of distilled water for 10 minutes to remove any $\mathrm{ZrO}_{2}$ residues. The crowns were then placed into the drying system (Robocam, Thermostar, Aachen, Germany) for 5 minutes at a temperature of $80^{\circ} \mathrm{C}$.

\section{ii) Sintering of Zirconia Crowns}

The crowns were then put into sintering furnace (Nabertherm, GmbH, Germany) and sintered at $1600^{\circ} \mathrm{C}$ for a total firing time of 12 hours as recommended by the manufacturer. Finally, all crowns were cleaned with distilled water for 5 minutes in ultrasonic cleaner (Skymen/OEM/ ODM, JP-031, Guangdong, China) to remove any contamination from the manufacturing process.

\section{Construction of Monolithic Vita Enamic Crowns:}

Vita Enamic hybrid ceramic was used in this study, it is composed of two parts; ceramic part (86 wt $\%$ / $75 \mathrm{vol} \%$ ) and polymer part (14 wt\% / 25 vol\%) 
- The ceramic part composition $(86$ wt $\%$ / 75 vol\%)

[Silicon dioxide $\mathrm{SiO}_{2}$ 58-63\%, Aluminum oxide $\mathrm{Al}_{2} \mathrm{O}_{3} 20-23 \%$, Sodium oxide $\mathrm{Na}_{2} \mathrm{O} 9-11 \%$, Potassium oxide $\mathrm{K}_{2} \mathrm{O} 4-6 \%$, Boron trioxide $\mathrm{B}_{2} \mathrm{O}_{3} 0.5-2 \%$, Zirconia $\mathrm{ZrO}_{2}<1 \%$, Calcium oxide $\mathrm{CaO}<1 \%$ ]

- The polymer part composition (14 wt\% / 25 vol\%)

UDMA (urethane dimethacrylate) TEGDMA (triethylene glycol dimethacrylate)

\section{Milling of Vita Enamic crowns:}

The milling procedure was carried out in the (vhf CAM 5-S1,camfacture AG, Germany) milling machine. After completion of the milling procedure, Vita Enamic crowns were finished with Sof-Lex discs (VITA Zahnfabrik, Germany) mounted into straight hand piece (NSK, Japan) and pre-polished with the pink polishers of the Vita Enamic Polishing kit (VITA Zahnfabrik, Germany) using water as a coolant and polished with the grey diamond-coated polishers of the Vita Enamic Polishing Set using slight pressure. Then all crowns were cleaned with distilled water for 5 minutes in the same ultrasonic cleaner.

\section{C-Testing procedure:}

\section{Vertical marginal gap distance:}

The prepared typodont molar tooth representing the patient's mouth was inserted in an acrylic resin mold. The tooth was protruding till $3 \mathrm{~mm}$ beyond the finish line. Then the vertical marginal gap distance was measured between each crown and prepared molar tooth without cementation. ${ }^{[28]}$ For standardization purposes, a holding jig was fabricated to secure the crowns on molar tooth to ensure the accuracy of their examination during vertical marginal gap measurement as carried out in different studies. ${ }^{[29]}$
Each crown was positioned onto Digital microscope with a built-in camera (Scope Capture Digital Microscope, Guangdong, China) at a magnification of $40 \mathrm{X}$. The images were captured and transferred to an IBM compatible personal computer equipped with the Image-Tool software (Vertical Image J 1.43U, National Institute of Health, USA) to measure and evaluate the gap of each crown. Shots of the margins were taken for each crown. Then morphometric measurements were done for each shot [4 equidistant landmarks] along the circumference for each surface of the crown (buccal, mesial, distal and lingual) 16 points per crown. Then the data obtained were collected, tabulated and then subjected to statistical analysis.

\section{Statistical Analysis}

One-way ANOVA followed by Tukey post hoc test was used to compare between more than two groups in non-related samples. Independent sample t-test was used to compare between two groups in non-related samples.

Two-way ANOVA was used to test the interaction between different variables.

Statistical analysis was performed with IBM (IBM Corporation, NY, USA) SPSS Statistics Version 20 for Windows (SPSS, Inc., IBM Company).

\section{RESULTS}

Data were presented as mean and standard deviation (SD) in $\mu \mathrm{m}$. Data were explored for normality by checking the data distribution and using Kolmogorov-Smirnov and Shapiro-Wilk tests. Vertical marginal gap data showed parametric distribution (Table 1)

Means with different small letters in the same column indicate statistically significance difference, means with different capital letters in the same row indicate statistically significance difference *; significant $(\mathrm{p}<0.05)$; non-significant $(\mathrm{p}>0.05)$ 
TABLE (1): Descriptive statistics for vertical marginal gap distance values of different groups and subgroups

\begin{tabular}{|c|c|c|c|c|c|}
\hline \multirow{3}{*}{ Variables } & \multicolumn{5}{|c|}{ Vertical marginal gap distance } \\
\hline & \multicolumn{2}{|c|}{ BruxZir crown } & \multicolumn{2}{|c|}{ Vita Enamic crown } & \multirow[t]{2}{*}{ p-value } \\
\hline & Mean & SD & Mean & SD & \\
\hline Intraoral scanning of typodont model & $42.34^{\mathrm{cA}}$ & 2.54 & $28.82^{\mathrm{cB}}$ & 2.24 & $<0.001 *$ \\
\hline Extraoral scanning of silicon impression & $48.48^{\mathrm{bA}}$ & 1.01 & $35.27^{\text {ьв }}$ & 4.17 & $<0.001^{*}$ \\
\hline Extraoral scanning of stone cast & $64.74^{\mathrm{aA}}$ & 3.32 & $44.81^{\mathrm{aB}}$ & 3.07 & $<0.001 *$ \\
\hline$p$-value & \multicolumn{2}{|c|}{$<0.001 *$} & \multicolumn{2}{|c|}{$<0.001 *$} & \\
\hline
\end{tabular}

\section{A) Effect of scanning techniques:}

The scanning techniques were statistically significantly affecting the vertical marginal gap distance of both crown materials at $\mathrm{P}$-value $<0.001$, it was graphically represented in Figure (4).

There was a statistically significant difference between (Intraoral scanning), (Extraoral scanning of silicon impression) and (Extraoral scanning of stone cast) where $(p<0.001)$. The lowest vertical marginal gap distance mean value was found in (Intraoral scanning) followed by (Extraoral scanning of silicon impression), while the highest mean value was found in (Extraoral scanning of stone cast)with both crown materials tested.

\section{B) Effect of crown material:}

The type of crown material was statistically significantly affecting the vertical marginal gap distance with the three scanning techniques (P-value $<0.001$ ), it was graphically represented in Figure (5).

There was a statistically significant difference between (BruxZir crowns) and (Vita Enamic crowns) where $(p<0.001)$ in all scanning techniques. Vita Enamic crowns had a lower statistically significant vertical marginal gap distance mean value than (BruxZir crown) with all tested scanning techniques.

\section{C) Interaction between all groups and sub-groups}

Two-way ANOVA analysis showed that the interaction between scanning techniques and crown materials on vertical marginal gap distance had a statistically significant effect at P-value (0.026; Table 2)

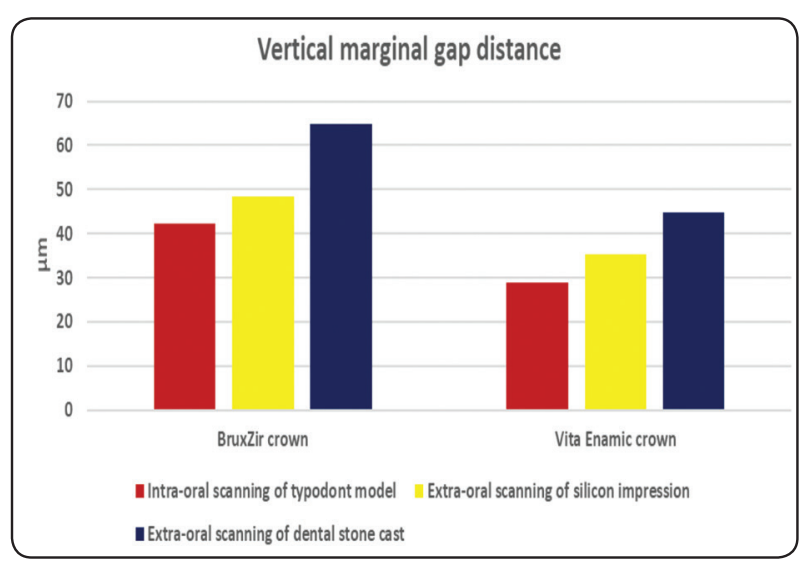

Fig. (4): Bar chart representing vertical marginal gap distance for different scanning techniques

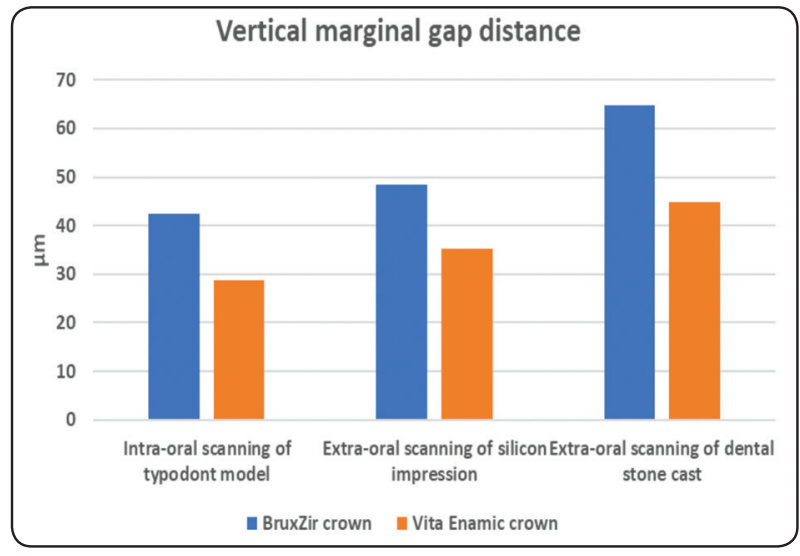

Fig. (5): Bar chart representing the effect of crown material on vertical marginal gap distance 
TABLE (2): Results of Two-way ANOVA for the effect of different scanning techniques and crown materials on vertical marginal gap distance.

\begin{tabular}{|c|c|c|c|c|c|}
\hline Source & $\begin{array}{c}\text { Type III Sum of } \\
\text { Squares }\end{array}$ & df & Means Square & F-value & P-value \\
\hline Corrected Model & $3801.021^{\mathrm{a}}$ & 5 & 760.204 & 90.604 & .000 \\
\hline Intercept & 58282.576 & 1 & 58282.576 & 6946.303 & .000 \\
\hline Scanning techniques & 1914.566 & 2 & 957.283 & 114.092 & .000 \\
\hline Crown materials & 1814.607 & 1 & 1814.607 & 216.271 & .000 \\
\hline Scanning techniques \&Crown materials & 71.847 & 2 & 35.924 & 4.282 & .026 \\
\hline Error & 201.371 & 24 & 8.390 & & \\
\hline Total & 62284.968 & 30 & & & \\
\hline Corrected Total & 4002.392 & 29 & & & \\
\hline
\end{tabular}

$d f:$ degrees of freedom $=(n-1), *$ Significant at $P<0.05$

\section{DISCUSSION}

After the introduction of CAD/CAM system for in-office fabrication of dental prosthesis (CEREC, Sirona Dental Systems St. Paul, MN, USA), remarkable changes have led to improvements in the quality of digital scans and subsequent restorations. Ting-Shu et al. (2015) ${ }^{[23]}$ informed that digital scanning provides distinct ascendancy in work efficiency and reduction in amount of materials, with the potential of further advancement and increasing use in the future. Digital impressions and scanning systems offer speed, efficiency, capability of storing information and transferring digital images between the dental office and the lab leading to better communication between them. ${ }^{[13]}$ Additionally, they have advantages as enhancing patient comfort, reducing the distortion of impression materials, $3 \mathrm{D}$ pre-visualization of tooth preparations by the operator and technician as it allow them to discuss any problem in the workflow. ${ }^{[30]}$

Execution of the methods of 3D digitization in dentistry is a modern and advanced substitute to the method of conventional impressions. Optical systems can be considered as most commonly used, particularly in fixed prosthodontics. The evolution of these systems have met up with two directions, extraoral and intraoral systems. The main quality parameters for assessing the measuring performances of 3D digitization methods and systems include precision and accuracy in addition to the operating effectiveness. ${ }^{[31]}$ Various digital impression systems are available, and each operates on a different principle. They include direct digitization intraorally or indirect digitization of gypsum cast. ${ }^{[11]}$ However, the accuracy of the indirect digitization by scanning the elastomeric impression has only been sparsely examined ${ }^{[27,32]}$ so the silicon impression scanning technique was chosen in this study.

Moreover, in this present study,10 silicon impressions were taken from the same typodont model and scanned then were poured to obtain 10 different stone casts to be scanned in order not only to assess the discrepancy due to the scan but also, to perform the same situation many times.

Several researches on the precision of digital impressions have been published, examining single-unit restorations, several teeth, quadrants, and full arch scans. ${ }^{[7,22]}$ A study by Lee et al. $(2013)^{[24]}$ proved the operator's preference of digital 
than conventional impression techniques. Gjelvold et al. (2016) $)^{[33]}$ reported that the assessments of dentists and patients have shown more comfort and less difficulty with digital impression techniques than conventional impression ones. Also, Wismeijer et al. (2013) $)^{[34]}$ concluded that patients significantly prefer digital scans as compared with material based conventional impression techniques.

The establishment of scanning techniques allows a great change in dental impression procedure and increases the accuracy of dental prostheses particularly in the marginal area due to the production of accurate final impression as any inaccuracy in impression results in restoration having marginal discrepancy that causes periodontal diseases, recurrent caries and subsequently restoration failure. ${ }^{[12]}$

The accuracy of digital impressions has been the purpose of many researches as it has been shown that the marginal adaptation is better in crowns obtained from digital impressions than in those obtained from conventional impressions. ${ }^{[9,23]}$ In an in vivo study, Syrek et al.(2010) $)^{[7]}$ obtained a lower marginal gap with intraoral digital impression than with conventional impression for zirconia crowns. Also, $\mathrm{Ng}$ et al.(2014) ${ }^{[10]}$ found vertical marginal gaps for digital impressions less than that for conventional impressions in lithium disilicate crowns.

Zirconia and hybrid ceramics copings have been proved to exhibit marginal and internal adaptation values within the clinically acceptable range. ${ }^{[35,36]}$ But there are few studies examining the marginal gap of recently introduced full-contour monolithic $\mathrm{CAD} / \mathrm{CAM}$ crowns as Zirconia and hybrid ceramics (Vita Enamic). ${ }^{[37,38]}$ So in this in vitro study, measuring vertical marginal gap distance of these monolithic ceramic materials which are both competent for posterior crowns were chosen.

Many requirements must be involved for proper function and durability of fixed prostheses, among them is the precision which is one of the most important factors for successful prostheses . One of the most common reasons for failure is inappropriate gap or imprecise restorations. In vivo studies have provided evidence that a large marginal discrepancy in a fixed restoration was usually accompanied with a higher plaque index and improper periodontal conditions. ${ }^{[12,39]}$

Marginal accuracy of the crowns in this study were calculated by measuring the vertical gap between the margin of tooth and that of the monolithic crown without cementation as when the crowns are cemented they may lose the accuracy of the primary adaptation by the influence of cement type, cement viscosity and cementation technique which might increase the marginal discrepancy. ${ }^{[28]}$

The null hypothesis for this in vitro study is rejected as there is a statistically significant differences in vertical marginal gap values between BruxZir and Vita Enamic crowns in the three scanning techniques.

The results of this study showed statistically significant differences between the three scanning techniques whereas the lowest vertical marginal gap distance was recorded with intraoral scanning followed by the extraoral scanning of silicon impression and the highest vertical marginal gap was found in the scanning of the stone cast group. This may be due to the elimination of impression and stone cast fabrication steps in case of intraoral scanning, leading to reduced technique sensitivity and more accurate dental prostheses with lower marginal gaps..$^{[7,27]}$ Additionally, the intraoral scanning technique used in this study was done on a typodont model which is more easier than intraoral scanning within the patient's mouth as the operator can move the scanner freely with more accessibility which is not the case intraorally with limited access, blood and saliva that could affect accuracy of final restoration. These results were consistent with Pedroche et al. (2016) ${ }^{[27]}$ who reported that the intraoral scanning technique has the lowest 
marginal gap value compared to the other groups (stone cast $\&$ impression scanning). Moreover, the present results were in agreement with Ahrberg et al. (2016) ${ }^{[40]}$ who found that the marginal gap values of zirconia crowns and three units FPDs were significantly lower with direct digitization than with indirect digitization.

The findings of this study were not coinciding with those of Persson et al.(2009) ${ }^{[41]}$ who compared the virtual images of scanning impressions and plaster models with virtual images obtained from scanning the master model, and concluded that there were no statistically significant differences between the results of the master model, the impression, and plaster groups. This contradiction may be attributed to the difference in the scanned teeth as they scanned canines, incisors, molars and found that the scanning of canines and incisors was more accurate while the scanning of molars presented the greatest discrepancies so they concluded that the form of dental preparation influences the results.

These current results were not in agreement with Luthardt et al.(2005) ${ }^{[42]}$ who informed that extraoral digitization of stone cast resulted in fabrication of dental prostheses with higher accuracy than intraoral scanning of the master model with confirmation that extraoral surface digitizing devices offer higher accuracy. This contrast might be due to the difference in the scanner type between the two studies as they used Cerec 3D. The results of present study were not coinciding with, Flugge et al. $(2013)^{[43]}$ who reported that data obtained by intraoral scanning was less precise than that obtained by extraoral digitization of stone models and that the intraoral scanning is affected by intraoral conditions such as saliva, blood, limited spacing, shape of preparation, and scanning position this might be attributed to the differences between the two studies as they performed an in vivo study and used a different scanner "iTero" which ensures that the results relied on the type of the scanner used.
In this research, extraoral scanning of silicon impression showed intermediate vertical marginal gap values that is statistically significantly higher than the intraoral scanning and lower than stone cast scanning. This might be attributed to that any conventional impression regardless of the material and technique is accompanied with an unavoidable degree of inaccuracy which is due to the number of steps and materials' manipulation as all impression materials are subjected to dimensional changes during setting which can affect marginal accuracy of final restoration. ${ }^{[23]}$ Additionally, in the silicon impressions there is difficulty in scan accuracy of certain areas that are difficult to access because of their anatomy and that may be hidden. ${ }^{[44]}$ So for these reasons the marginal gap values of intraoral scanning technique is less than impression scanning one which was in agreement with Pedroche et al.(2016) ${ }^{[27]}$ who reported that intraoral scanning had lower marginal gap than extraoral scans of polyvinyl siloxane impressions. Also, this result was consistent with Bosniac et al.(2019) ${ }^{[32]}$ who found that the intraoral scanning promote the production of single-tooth zirconia restorations with an adequate marginal fit while impression scanning led to the production of restorations with considerably higher marginal gap values.

However, the vertical marginal gap values of the silicon impression scanning was lower than those of scanning stone cast as for cast scanning additional steps over those employed in impression scanning were done. Therefore, silicon impression scanning is a technique of reducing the manufacturing process as the step of pouring dental stone into the impression is eliminated.A previous study evaluating the accuracy of scanned data from impressions and stone casts reported that eliminating the stone cast fabrication stage can improve process efficiency. ${ }^{[4]}$ As the stone cast fabrication is a multifactorial process that found to be dependent on many factors including type of dental stone, the water/ powder ratio, stone mixing technique, its compatibility with impression materials and degree of expansion during setting of 
stone which might affect the marginal accuracy of the final crown. ${ }^{[45]}$ Additionally, the application of non-aqueous developer on the silicon impressions before scanning as it reduce brightness and improve reproduction of all details including the finish line might affect marginal accuracy of final restoration obtained from silicon impression scanning. ${ }^{\text {[27] }}$

These current results were consistent with Pedroche et al.(2016) [27] who reported that impression scanning technique has lower marginal gap value compared to stone cast group. But the results of the present study were not in agreement with Lee et al.(2014) ${ }^{[46]}$ who tested the marginal gaps of two groups of prostheses produced from scanning stone cast and another group from scanning silicone impressions, gaps were found to be higher in the silicone impression scanning group. They informed that this result may have been due to the milling instruments used; however, the limited resolution of the scanning system for the digitization of the concavity, the length and the diameter of the negative abutment formed in the impression, and the color of the impression material may have influenced the quality of the scanned impression. The findings of our study were different from the study of Aranda Yus et al.(2018) $)^{[44]}$ who determined the marginal discrepancy in monolithic zirconia crowns made by comparing scans of silicone impressions and of stone casts and found significant differences with higher marginal gaps in the impression scanning group and related their results to that there was a difficulty in scan accuracy of certain surfaces that are difficult to access in the silicon impressions and that relief forms are the most accurate for obtaining images, either through intraoral scanning or extraoral scanning of stone models.

Results showed that the marginal gap values of Vita Enamic crowns were statistically significantly lower than that of BruxZir crowns with the different tested scanning techniques. This may be due the differences in the chemical composition and the post-milling treatment between Vita Enamic and
BruxZir where Vita Enamic obtains its physical properties directly after milling as no firing process is required according to their manufacturer's instructions. Vita Enamic crowns were only polished after milling without exposure to a sintering cycle at high temperature as for BruxZir crowns which may help to ensure a high degree of dimensional accuracy for the final Vita Enamic restorations. Additionally, the higher marginal gap obtained in BruxZir crowns might be due to sintering shrinkage of thick zirconia material of the full contour monolithic zirconia crowns as the monolithic zirconia crowns are thicker than zirconia cores, a fact that may cause significantly more sintering contraction. [37] This finding was in agreement with Majeed \& Al-Adel (2016) ${ }^{[37]}$ and Güngör et al. (2018) ${ }^{[38]}$ who found that full contour CAD/CAM crowns made from hybrid ceramics showed better marginal and internal fitness than the zirconia crowns.

There is a controversy in dental literature regarding the clinically acceptable marginal gap, but many literatures reported that a marginal gap below $120 \mu \mathrm{m}$ is considered as clinically acceptable as Borba et al. (2013) $)^{[4]}$ and Al-Zubaidi et al. (2015) ${ }^{[48]}$ However, the acceptable marginal gap for CAD/CAM restorations was approved to be less than $90 \mu \mathrm{m} .{ }^{[49]}$ Therefore, the marginal gap values observed in this study for all the tested monolithic crowns are all within the clinically acceptable range.

There are some limitations of this study; as the typodont acrylic resin tooth used showed a lightreflection and light transmission differ from the natural dentition. Also, the impression was taken under in vitro conditions that differ greatly from impression taken in the oral cavity. Moreover, the intraoral scanning technique used in this research is different from the real conditions due to the presence of saliva and limited access for the scanner intraorally. So further research is necessary to validate the presented results under clinical conditions. 


\section{CONCLUSIONS}

Within the limitations of the present study the following conclusions can be drawn:

1- Vertical marginal gap distance of monolithic crowns was significantly affected by the scanning techniques and crown materials with the superiority of the intraoral scanning technique.

2- Vita Enamic crowns had lower vertical marginal gap distance than BruxZir crowns with different scanning techniques.

3- All the tested monolithic crowns showed marginal gap distance values within the clinically acceptable levels.

\section{REFERENCES:}

1- Daou EE. The zirconia ceramic: strengths and weaknesses. Open Dent J $2014 ; 8: 33-42$.

2- Datla SR, Alla RK, Alluri VR, J. B. P, Konakanchi A. Dental Ceramics : Part II - Recent Advances in Dental Ceramics. Am J of Mat Eng and Techn 2015; 3(2):19-26.

3- De C. Ramos N, Campos TM, De La Paz IS, Machado J P , Bottino MA, Cesar PF, De Melo R M . Microstructure characterization and SCG of newly engineered dental ceramics. Dent Mater 2016 ;32(7): 870-878.

4- Min J, Arola DD, Yu D, Yu P, Zhang Q, Yu H, Gao S. Comparison of human enamel and polymer-infiltrated-ceramic-network material 'ENAMIC' through micro- and nano-mechanical testing. Ceram Int 2016;42(9):10631-10637.

5- Zhang Y, Lee JJ, Srikanth R. Edge chipping and flexural resistance of monolithic ceramics. Dent Mater 2013; 29:1201-1208

6- Baldassarri M, Stappert CF, Wolff MS. Residual stresses in porcelain-veneered zirconia prostheses. Dent Mater 2012;28:873-879.

7- Syrek A, Reich G, Ranftl D. Clinical evaluation of allceramic crowns fabricated from intraoral digital impressions based on the principle of active wavefront sampling. J Dent 2010;38:553-559.

8- Kokubo Y, Nagayama Y, Tsumita M. Clinical marginal and internal gaps of In-Ceram crowns fabricated using the GN-I system. J Oral Rehabil 2005;32:753-758.
9- Pradies G, Zarauz C, Valverde A. Clinical evaluation comparing the fit of all-ceramic crowns obtained from silicone and digital intraoral impressions based on wavefront sampling technology. J Dent 2015;43:201-208.

10- $\mathrm{Ng} \mathrm{J}$, Ruse D, Wyatt C. A comparison of the marginal fit of crowns fabricated with digital and conventional methods. J Prosthet Dent 2014;112:555-560.

11- Logozzo S, Franceschini G, Kilpel“a A. A comparative analysis of intraoral 3D digital scanners for restorative dentistry. Internet J Med Technol 2011;5(1):1-19.

12- Birnbaum NS, Aaronson HB, Stevens C, Cohen B. 3D Digital Scanners: A High-Tech Approach to More Accurate Dental Impressions. J Inside Dentistry 2009; 5(4):1-12.

13- Kim SY, Kim MJ, Han JS, Yeo IS ,Lim YJ, Kwon HB. Accuracy of dies captured by an intraoral digital impression system using parallel confocal imaging. Int J Prosthodont 2013;26(2):161-163.

14- De Paula Silveira AC, Chaves SB, Hilgert LA, Ribeiro AP. Marginal and internal fit of CAD-CAM-fabricated composite resin and ceramic crowns scanned by 2 intraoral cameras. J Prosthet Dent 2017; 117(3): 386-392.

15- Ziegler M. Digital impression taking with reproducibly high precision. Int J Comput Dent 2009; 12: 159-163.

16- Neves Flavio D. Micro-computed tomography evaluation of marginal fit of lithium disilicate crowns fabricated by using chairside CAD/CAM systems or the heat-pressing technique. J prosthet Dent 2014;112(5):1134-1140.

17- Boitelle P, Mawussi B, Tapie L, Fromentin O. A systematic review of CAD/CAM fit restoration evaluations. J Oral Rehabil 2014;41:853-874.

18- Zeltner M, Sailer I, Muhlemann S, Ozcan M, Hammerle $\mathrm{CH}$, Benic GI. Randomized controlled within -subject evaluation of digital and conventional workflows for the fabrication of lithium disilicate single crowns. Part III: marginal and internal fit .J Prosthet Dent 2017;117:354362.

19- Berrendero S, Salido MP, Valverde A, Ferreiroa A, Pradies G. Influence of conventional and digital intraoral impressions on the fit of CAD/CAM-fabricated all-ceramic crowns. Clin Oral Investig 2016;20:2403-2410.

20- Su TS and Sun J. Comparison of marginal and internal fit of 3-unit ceramic fixed dental prostheses made with either a conventional or digital impression. J Prosthet Dent 2016;116:362-367. 
21- Ueda K, Beuer F, Stimmelmayr M, Erdelt K, Keul C, Guth JF. Fit of 4-unit FDPs from $\mathrm{CoCr}$ and zirconia after conventional and digital impressions. Clin Oral Investig 2016;20:283-289.

22- Brawek PK, Wolfart S, Endres L, Kirsten A, Reich S. The clinical accuracy of single crowns exclusively fabricated by digital workflow the comparison of two systems. Clin Oral Investig 2013, 17(9):2119-2125.

23- Ting-Shu S , Jian S. Intraoral digital impression technique: a review. J of Prosthodont 2015; 24(4): 313-321.

24- Lee SJ, Macarthur RX 4th, Gallucci GO. An evaluation of student and clinician perception of digital and conventional implant impressions. J Prosthet Dent 2013;110(5): 420-423.

25- Yuzbasioglu E, Kurt H, Turunc R, Bilir H.Comparison of digital and conventional impression techniques: evaluation of patients' perception, treatment comfort, effectiveness and clinical outcomes. BMC Oral Health. $2014 ; 14: 10$.

26- Praca L, Pekam FC, Rego RO, Radermarcher K ,Wolfart S, Marotti J. Accuracy of single crowns fabricated from ultrasound digital impressions. Dent Mater 2018;34:80-88

27- Pedroche LO, Bernardes SR, Leão MP, Kintopp CC, Correr GM, Ornaghi BP, Gonaza CC. Marginal and internal fit of zirconia copings obtained using different digital scanning methods. Braz Oral Res.2016; 30(1):e113.

28- Renne W, McGill ST, Forshee KV, DeFee MR, Mennito AS. Predicting marginal fit of CAD/CAM crowns based on the presence or absence of common preparation errors. J Prosthet Dent 2012;108:310-315.

29- Thiab SS ,Zakaria MR. The evaluation of vertical marginal discrepancy induced by using as cast and as received base metal alloys with different mixing ratios for the construction of porcelain fused to metal copings. Al-Rafidain Dent J 2004;4(1)10-19.

30- Christensen GJ. Impressions are changing: deciding on conventional, digital or digital plus in-office milling. JADA 2009, 140:1301-1304.

31- Vlaar ST, Van der Zel JM. Accuracy of dental digitizers. Int Dent J 2006; 56 :301-309.

32- Bosniac P, Rehmann P, Wostmann B. Comparison of an indirect impression scanning system and two direct intraoral scanning systems in vivo. Clin oral invest 2019; 23(5):2421-2427.
33- Gjelvold B, Chrcanovic BR, Korduner EK, Collin-Bagewitz I, Kisch J. Intraoral digital impression technique compared to conventional impression technique. A randomized clinical trial. J Prosthodont 2016;25:282-287.

34- Wismeijer D, Mans R, van Genuchten M, Reijers HA. Patients' preferences when comparing analogue implant impressions using a polyether impression material versus digital impressions (Intraoral Scan) of dental implants. Clinical Oral Implants Research 2013; 25(10):1113-1118.

35- Lins L, Bemfica V, Queiroz C, Canabarro A. In vitro evaluation of the internal and marginal misfit of CAD/CAM zirconia copings. J Prosthet Dent 2015;113:205-211.

36- Azarbal A, Azarbal M, Engelmeier RL, Kunkel TC. Marginal Fit Comparison of CAD/CAM Crowns Milled from Two Different Materials. Journal Prosthodont 2018 ; 27(5):421-428.

37- Majeed MA, Al-Adel SK. Evaluation of the marginal and internal fitness of full contour CAD/CAM crowns made from zirconia, lithium disilicate, zirconia-reinforced lithium silicate and hybrid dental ceramic by silicone replica technique (A comparative In vitro study) J Genet Environ Resour Conserv 2016; 4(1):10-20.

38- Güngör MB, Doğan A, Bal BT, Nemli SK. Evaluation of marginal and internal adaptations of posterior all-ceramic crowns fabricated with chair-side CAD/CAM system: an in vitro study. Acta Odontol Turc 2018;35(1):1-8.

39- Sailer I, Makarov NA, Thoma DS, Zwahlen M, Pjetursson BE. All-ceramic or metal-ceramic tooth-supported fixed dental prostheses (FDPs)? A systematic review of the survival and complication rates. Part I: single crowns (SCs). Dent Mater 2015;31:603-623.

40- Ahrberg D, Lauer HC, Ahrberg M, Weigl P. Evaluation of fit and efficiency of CAD/CAM fabricated all-ceramic restorations based on direct and indirect digitalization: a double-blinded, randomized clinical trial. Clin Oral Investig 2016; 20: 291-300.

41- Persson AS, Odén A, Andersson M, Sandborgh-Englund G. Digitization of simulated clinical dental impressions: virtual three-dimensional analysis of exactness. Dent Mater 2009; 25:929-936.

42- Luthardt RG, Loos R, Quaas S. Accuracy of intraoral data acquisition in comparison to the conventional impression. Int J Comput Dent 2005; 8:283-294. 
43- Flügge TV, Schlager S, Nelson K, Nahles S, Metzger MC. Precision of intraoral digital dental impressions with iTero and extraoral digitization with the iTero and a model scanner. Am J Orthod Dentofacial Orthop 2013;144(3):471-478.

44- Aranda Yus E, Cantarell JMA, Miñarro Alonso A . Comparison of the marginal fit of milled yttrium stabilized zirconium dioxide crowns obtained by scanning silicone impressions and by scanning stone replicas. J Adv Prosthodont 2018;10:236-244.

45- Powers J.Gypsum products and investments. In: Craig's Restorative Dental Materials. Edited by Powers J. St Louis: Mosby, 2006:313-336.

46- Lee WS, Kim WC, Kim HY, Kim WT, Kim JH. Evaluation of different approaches for using a laser scanner in digi- tization of dental impressions. J Adv Prosthodont 2014; 6:22-29.

47- Borba M, Miranda WG Jr, Cesar PF, Griggs JA, Bona AD. Evaluation of the adaptation of zirconia-based fixed partial dentures using micro-CT technology. J Braz Oral Res 2013; 27 (5):396-402.

48- Al-Zubaidi ZAK, Al-Shamma AMW. The effect of different finishing lines on the marginal fitness of full contour zirconia and glass ceramic CAD/CAM crowns (An in vitro study). J. Dent Mater Tech 2015; 4(3): 127-136.

49- Memari Y, Mohajerfar M, Armin A, Kamalian F, Rezayani V, Beyabanaki E. Marginal adaptation of CAD/CAM all ceramic crowns made by different impression methods : A literature Review. Journal Prosthodont 2019;28(2):95-221. 